location was shifted in the anterior-posterior direction $( \pm 5 \%$ and $\pm 10 \%)$ were significantly associated with joint moment and power values $(\mathrm{p}<0.01)$. The joint moment and power are more sensitive to errors in the CoP calculated from a force plate measurements than in BSP parameters. Consequently, those results suggest that it is necessary to pay close attention to the level of experimental errors to ensure meaningful results when using inverse dynamics procedures in various fields.

\section{A 6-YEAR RETROSPECTIVE REVIEW OF INJURIES SUSTAINED DURING THE SINGAPORE CRICKET CLUB INTERNATIONAL RUGBY SEVENS TOURNAMENT}

${ }^{1} \mathrm{C} \mathrm{Xu},{ }^{2} \mathrm{~J}$ Walter, ${ }^{3} \mathrm{LL}$ Low, ${ }^{4} \mathrm{KW}$ Lai. ${ }^{1}$ Department of Family Medicine, Singhealth Polyclinics, Singapore; ${ }^{2}$ Singapore Cricket Club, Singapore; ${ }^{3}$ Department of Family Medicine and Continuing Care, Singapore General Hospital, Singapore; ${ }^{4}$ sland Orthopaedic Consultants, Singapore

\subsection{6/bjsports-2018-ISSSMC2018.4}

Rugby Sevens is gaining popularity in Asia as evidenced by the increase in number of tournaments and participants of the sport. Currently, there are limited studies that look at injury statistics for Rugby Sevens, especially at the amateur level. This study aims to assess injury patterns among amateur Rugby Sevens players participating in the annual Singapore Cricket Club Rugby Sevens International tournament from 2012 to 2017. A retrospective review was made of recorded injury data of all players participating in the 2012 to 2017 Singapore Cricket Club Rugby Sevens Internationals tournament. Main outcome measures include incidence rate of injuries expressed per 1000 player hours, injury rate according to anatomical location, and comparative injury incidence between successive days within each tournament. 343 injuries were recorded over the 6 tournaments, with an injury incidence of 348 per 1000 player hours. The lower limb was the most commonly injured region (46\%, 159 per 1000 paying hours), followed by head and neck injuries (24\%, 82 per 1000 playing hours), upper limb injuries (21\%, 74 per 1000 playing hours) and trunk injuries (9\%, 32 per 1000 playing hours). There was a greater incidence of injuries on day 3 of competition compared to day 1 for the 2013 and 2016 tournaments (2013: 541 per 1000 player hours vs. 520 per 1000 player hours; 2016: 191 per 1000 player hours vs. 767 per 1000 player hours). Being the first study of injuries in Asian Rugby Sevens, this serves to inform of the background risk of injuries, which is much higher than is currently reported in the literature. A well-designed, prospective injury surveillance study will be necessary to investigate if injury rates are indeed higher at the amateur level in Asia, and whether there are modifiable risk factors unique to this part of the world which should be considered to guide injury prevention programmes.

\section{\begin{tabular}{|l|l}
5 & THE INTERCHANGEABILITY OF THE 2.4 KM RUN TIME
\end{tabular} AND MULTISTAGE FITNESS TEST SCORE AS PERFORMANCE PREDICTORS FOR PHASE 2 ARMY RECRUITS COMPLETING A 12.8 KM LOADED MARCH CARRYING AN 11 KG BACKPACK AND 4 KG RIFLE}

D Moore, C Harrison, S Moore. School of Social Sciences, Humanities and Law, Teesside University, UK

\subsection{6/bjsports-2018-ISSSMC2018.5}

Pre-loaded-march fitness tests are implemented continually during a soldier's career. The $2.4 \mathrm{~km}$ maximal-effort run protocol and the multistage fitness test (MSFT) protocol are used interchangeably as a surrogate tests prior to a loaded-march. Previous research identified that the $2.4 \mathrm{~km}$ run time and MSFT score have a strong correlation, no research examined if they had a similar predictive strength in relation to loaded-march performance. This study aimed to quantify the predictive strength of the MSFT score and $2.4 \mathrm{~km}$ maximal-effort run time for performance in a $12.8 \mathrm{~km} 15 \mathrm{~kg}$ loaded-march and if the MSFT and $2.4 \mathrm{~km}$ maximal-effort run can be used interchangeably with the same predictive strength alongside strength tests and anthropometric measurements from current Army protocols. Testing was completed over eight-days with phase-two British Army recruits from the Royal Electrical and Mechanical Engineers (REME) ( $\mathrm{n}=12$ male recruits aged 1827). Day one involved collection of anthropometric data and completion of the $2.4 \mathrm{~km}$ run protocol, on day four the MSFT protocol was completed and on day eight the $12.8 \mathrm{~km}$ $15 \mathrm{~kg}$ loaded-march protocol was completed with heart-rate (HR) recorded throughout. The MSFT score and $2.4 \mathrm{~km}$ maximal-effort run time do not adequately predict the average percentage of estimated HR maximum (average\%E-HRmax) during a $12.8 \mathrm{~km} 15 \mathrm{~kg}$ loaded-march $(\mathrm{p}=-470$ and 0.513 respectively). The MSFT score alongside measures of waist circumference (WC), body fat percentage (BF\%) and height is a strong predictor of average\%E-HRmax during the $12.8 \mathrm{~km}$ $15 \mathrm{~kg}$ loaded-march $(\mathrm{r}=-0.668, \mathrm{p}=0.049)$. The MSFT score, WC, weight and BF\% showed a statistically significant negative correlation with the average $\% \mathrm{E}-\mathrm{HRmax}$ during the $12.8 \mathrm{~km} 15 \mathrm{~kg}$ loaded march $(\mathrm{r}=-0.794, \mathrm{p}=0.011)$, the $2.4 \mathrm{~km}$ run time, WC, weight and $\mathrm{BF} \%$ exhibited a statistically significant strong positive correlation in relation to the average \%E-HRmax during the $12.8 \mathrm{~km} 15 \mathrm{~kg}$ loaded-march $(\mathrm{r}=0.726, \mathrm{p}=0.027)$. The MSFT and the $2.4 \mathrm{~km}$ maximaleffort run can be used interchangeably alongside measures of $\mathrm{BF} \%$, WC and weight to predict $12.8 \mathrm{~km} 15 \mathrm{~kg}$ loaded-march performance. Low $\mathrm{BF} \%$ and $\mathrm{WC}$ suggest that the presence of excess adipose tissue is detrimental to performance during load-carriage. The current Army strength tests have limited significance to load-carriage performance.

\section{\begin{tabular}{|l|l}
\hline 6 & THE LANDING ERROR SCORING SYSTEM (LESS) AND
\end{tabular} LOWER LIMB POWER PROFILES IN ELITE RUGBY UNION PLAYERS}

${ }^{1} S$ Rowell, ${ }^{1,2} \mathrm{~N}$ Relph. ${ }^{1}$ Department of Medical and Sports Sciences, University of Cumbria, UK; ${ }^{2}$ Department of Applied Health and Social Care, Edge Hill University, UK

\subsection{6/bjsports-2018-ISSSMC2018.6}

The Landing Error Scoring System (LESS) is a relatively new clinical test that assesses landing biomechanics during a drop jump task. Performance measures such as Jump Height (JH), Power (P), Contact time (CT) and Reactive Strength Index (RSI) are common performance measures in an athletic population. Comparing results from the LESS against these performance measures has not previously been reported in an elite rugby union setting. The aim of this study was to compare differences between LESS scores and lower limb performance measures in elite male rugby union players. Thirty two male, elite rugby union players participated in the study. Each participant completed 3 trials of the LESS. Performance data 
regarding $\mathrm{JH}, \mathrm{P}, \mathrm{CT}$ and RSI were recorded concurrently via the OptoJump system (Microgate, Bolzano, Italy). LESS trials were scored independently by the authors. Statistical analyses were used to confirm inter and intra-rater reliability. Data was tested for normality and one-way factorial MANOVA was used to assess between group differences $(p<0.05)$. Ethical approval was granted by the University's Ethics Committee. Intra-class Correlation Coefficients (ICC) demonstrated excellent intra (0.96) and inter (0.94) rater reliability for the LESS in the current study. Six participants produced LESS scores deemed excellent to good (score $\geq 6$ ), 26 participants produced scores deemed moderate to poor (score $\leq 5$ ). Participants with moderate to poor LESS scores produced significantly greater P $\quad(p=0.038)$, RSI $(p=0.016)$ and lower CT $(p=0.002)$, there was no significant difference in $\mathrm{JH}$ $(p=0.842)$ between participants scoring excellent to good and moderate to poor. The current study reports excellent intra and inter rater reliability for the LESS, supporting its use as a clinical assessment tool in an elite rugby union populations. The majority of players presented with moderate to poor LESS scores, therefore landing biomechanics may need to be improved in this population. Participants scoring moderate to poor in the LESS recorded significantly higher P, CT and RSI but not JH. This suggests participants with high risk landing biomechanics may also produce higher performance measures, but these do not result in improved outcome performance such as jump height.

\section{SUNDAY STRATEGY AFFECTS MATCH DAY +2 SALIVARY CORTISOL AND SIGA RESPONSE FOLLOWING SATURDAY MATCHES THROUGH AN ENGLISH CHAMPIONSHIP FOOTBALL CLUB SEASON}

1) Dunbar, ${ }^{2} \mathrm{M}$ King, ${ }^{2} \mathrm{C}$ Haslam, ${ }^{3,4} \mathrm{~A}$ Nevill, ${ }^{3,5} \mathrm{G}$ Warrington. ${ }^{1}$ Soma Bioscience Limited, Wallingford, UK; ${ }^{2}$ Brentford Football Club, Brentford, UK; ${ }^{3}$ Department of Physical Education and Sport Sciences, University of Limerick, Ireland; ${ }^{4}$ Institute of Sport and Human Science, University of Wolverhampton, UK; ${ }^{5}$ Health Research Institute, University of Limerick, Ireland

\subsection{6/bjsports-2018-ISSSMC2018.7}

The measurement of salivary biomarkers has become popular in professional sport, in an attempt to monitor the stress responses associated with training, competition, and other related lifestyle factors. Using a 'Point of Care' platform, giving results within minutes of sample collection in the professional football environment, the aim is to provide coaching staff with 'readiness to train' data relating to individual players or the squad as a whole. At this club, after a Saturday match where there is no game until the following Saturday, players do not report to training until Monday Morning. However, if the next game is midweek, players are required to report to training on Sunday morning for recovery protocols. Salivary samples were collected for evaluation of sIgA and cortisol from 26 players (age $24.1 \pm 2.9$ y, body mass 78.7 $\pm 6.5 \mathrm{~kg}$, stature $1.81 \pm 0.07$ ) in a Championship football club squad at 09:00 on the Monday following a Saturday Match throughout the 2017-2018 season. In total 19 time-points were analysed; 11 where players reported in on a Sunday and 8 where players had the Sunday off from training post-match. All saliva samples were analysed at the training ground using Soma dual sIgA/Cortisol LFDs read with a Soma Cube LFD Reader to give rapid quantitative values for sIgA and cortisol.
sIgA was seen to be variable, both within (CV 53.8\%) and between players (CV 62.1\%), as was the cortisol response (within CV 53.2\% and between players CV 65.3\%). MultiLevel regression analysis revealed a highly significant quadratic effect in sIgA due to 'weeks' throughout the season (the intercept at zero weeks was $187 \mu \mathrm{g} / \mathrm{mL}$ initially increasing at the early weeks, peaking mid-season then declining significantly towards the end of the season). Where players reported to training on Sunday, the Monday sIgA response was $60 \mu \mathrm{g} / \mathrm{mL}$ lower than when Sundays were spent at home. The Model for cortisol response showed a highly significant linear increase throughout the season. However, the impact of reporting for recovery training on Sunday had a significant impact on Monday cortisol, changing from 4.9 (0.8) nM when they did report in on Sunday to 9.6 (0.6) nM when they did not. Such biomarker responses may have important implications and practical applications for the planning of recovery strategies for subsequent professional football matches.

\section{THE SALBUTAMOL PASSPORT: HOW TO RULE OUT AN ADVERSE ANALYTICAL FINDING FROM SERIAL URINE TESTS}

${ }^{1}$ Daren Austin, ${ }^{2}$ Morten Hostrup, ${ }^{3}$ Sheila M Bird. 'Senior Fellow and Senior Director, Clinical Pharmacology, GlaxoSmithKline; '2Associate Professor, Section of Integrative Physiology, Department of Nutrition, Exercise and Sports, University of Copenhagen; ${ }^{3}$ MRC Biostatistics Unit, University of Cambridge Institute for Public Health

\subsection{6/bjsports-2018-ISSSMC2018.8}

Salbutamol is used widely by elite athletes in treatment of asthma and related conditions, such as exercise-induced bronchoconstriction. In competitive sport, salbutamol is permitted by inhalation at doses up to $1600 \mu \mathrm{g} / \mathrm{day}$, not to exceed $800 \mu \mathrm{g}$ in any 12 hour period. WADA has established a urinary salbutamol Decision Limit for a presumed adverse analytical finding (AAF) of $1200 \mathrm{ng} / \mathrm{mL}$. Urine salbutamol levels greater than this are deemed to be a result of prohibited use or excessive supratherapeutic inhalation. Studies have shown that under dehydrated conditions, exercise increases the risk of exceeding the Decision Limit after single inhaled doses of $1600 \mu \mathrm{g}$. One means of explaining an AAF is a Pharmacokinetic (PK) Study to establish whether an athlete is an outlier with higher urine concentrations than typical. However, a recent salbutamol case from the 2017 Vuelta a España, deemed it impractical and impossible to conduct a valid PK study capable of recreating the complex conditions of an athlete competing in such an event. Another means of explanation is the 'Salbutamol Passport'. This is a statistical model derived from serial test data obtained from an event during periods of stable salbutamol use. After adjustment for dose and dosing frequency, the Salbutamol Passport can be used to predict an expected concentration range for days when an athlete might increase their salbutamol intake due to worsening symptoms. Since the number of tests during stable use is typically small, the method uses propagation of uncertainty in mean and variance of the log-transformed salbutamol concentrations to derive expected bands for any dose and frequency of interest. The method is demonstrated with data from a cyclist competing in the 2007 Giro d'Italia. The athlete provided four salbutamol tests whilst taking $400 \mathrm{ug} /$ day with geometric mean $461 \mathrm{ng} / \mathrm{mL}$ (95\% CL: 352-604) and one test of 